\title{
Isolation of neural precursor cells from skeletal muscle tissues and their differentiation into neuron-like cells
}

\author{
Jung Sik Park', Soyeon Kim², \\ Dong Keun Han ${ }^{3}$, Ji Youl Lee 4 \\ and Sung Ho Ghil ${ }^{1,5}$ \\ ${ }^{1}$ Department of Life Science, Kyonggi University \\ Suwon 443-760, Korea \\ ${ }^{2}$ Department of Anatomy \\ School of Medicine, Ajou University \\ Suwon 443-749, Korea \\ ${ }^{3}$ Biomaterials Research Center \\ Korea Institute of Science and Technology \\ Seoul 130-650, Korea \\ ${ }^{4}$ Department of Urology \\ College of Medicine, The Catholic University of Korea \\ Seoul 137-701, Korea \\ ${ }^{5}$ Corresponding author: Tel, 82-31-249-9646; \\ Fax, 82-31-249-9646; E-mail, shghil@ kyonggi.ac.kr
}

Accepted 22 May 2007

Abbreviations: bHLH, basic helix-loop-helix; FSK, forskolin; HSC, hematopoietic stem cell; MDSCs, muscle-derived stem cells; MSC, mesenchymal stem cell; NSC, neural stem cell; NSE, neuron-specific enolase; PM, proliferation medium; RA, retinoic acid; RT, reverse transcriptase; SMNPs, skeletal muscle-derived neural precursor cells

\begin{abstract}
Skeletal muscle contains several precursor cells that generate muscle, bone, cartilage and blood cells. Although there are reports that skeletal muscle-derived cells can trans-differentiate into neural-lineage cells, methods for isolating precursor cells, and procedures for successful neural induction have not been fully established. Here, we show that the preplate cell isolation method, which separates cells based on their adhesion characteristics, permits separation of cells possessing neural precursor characteristics from other cells of skeletal muscle tissues. We term these isolated cells skeletal muscle-derived neural precursor cells (SMNPs). The isolated SMNPs constitutively expressed neural stem cell markers. In addition, we describe effective neural induction materials permitting the neuron-like cell differentiation of SMNPs. Treatment with retinoic acid or forskolin facilitated morphological changes in SMNPs; they differentiated into neuron-like cells that possessed specific neuronal markers. These
\end{abstract}

results suggest that the preplate isolation method, and treatment with retinoic acid or forskolin, may provide vital assistance in the use of SMNPs in cell-based therapy of neuronal disease.

Keywords: cell differentiation; muscle, skeletal; neuroD protein; stem cells; tissue therapy

\section{Introduction}

Recent studies have shown that adult stem cells can differentiate into various types of cells. For instance, mesenchymal stem cells (MSC) can differentiate into blood, myogenic, vascular or neurogenic cells (Jiang et al., 2002; Muguruma et al., 2003; Kim et al., 2005); skin stem cells can differentiate into neurons, smooth muscle cells or adipose cells (Toma et al., 2001, 2005) and adipose stem cells can create osteogenic, myogenic, or chondrogenic lineages (Zuk et al., 2002; Lin et al., 2005). These findings indicate that adult stem cells have the capability to differentiate into tissues other than the tissues from which they originated. Recently, it has been reported that skeletal muscle-derived cells can also generate multiple cell types, characteristic of various tissues and organs. The pluripotentiality of skeletal muscle-derived cells seems to be greater than was once thought (McKinney-Freeman et al., 2002; Wada et al., 2002; Peng and Huard, 2004; Hwang et al., 2004; Sun et al., 2005). It is of particular interest that skeletal muscle-derived cells can trans-differentiate into neuron-like cells (RomeroRamos et al., 2002; Alessandri et al., 2004; Kondo et al., 2006). The methods of isolation of precursor cells, and the procedures for successful neural induction, have not, however, been fully established. In this study, we describe cell isolation methods allowing for the separation of cells that possessed neural precursor characteristics from skeletal muscle-derived cells. We term these cells "skeletal muscle-derived neural precursor cells (SMNPs)". We also report on materials permitting effective neural induction of SMNPs. 
A

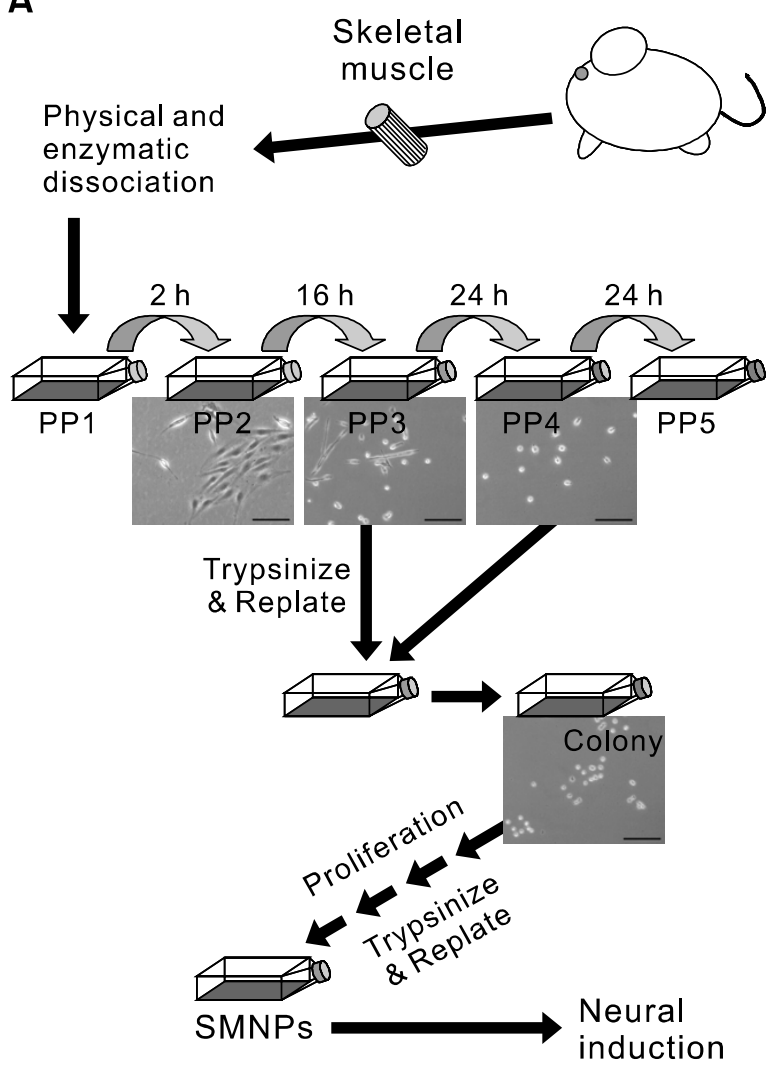

B

\begin{tabular}{|c|cc|}
\multicolumn{1}{c}{$6 \mathrm{~d}$} & $2-3$ weeks & $5 \mathrm{~d}$ \\
\hline $\begin{array}{c}\text { Isolation } \\
\text { (Preplate } \\
\text { method) }\end{array}$ & $\begin{array}{c}\text { Proliferation } \\
\text { (Trypsinize and replate) }\end{array}$ & $\begin{array}{c}\text { Neural } \\
\text { induction }\end{array}$ \\
\hline & \multicolumn{2}{c|}{$\begin{array}{r}\text { Characterization } \\
\text { (Figure 2) } \\
\text { Characterization } \\
\text { (Figure 3 and 4) }\end{array}$} \\
\end{tabular}

Figure 1. Isolation and proliferation of skeletal muscle-derived neural precursor cells (SMNPs). (A) A preplate isolation method was used to obtain SMNPs as described in the Materials and Methods section. The morphological phenotypes of each passage flask are represented. Scale bar: $50 \mu \mathrm{m}$. (B) Timetable of isolation and proliferation of SMNPs.

\section{Materials and Methods}

\section{Isolation and proliferation of SMNPs}

Primary skeletal muscle cultures were prepared from 3-week-old ICR mice. SMNPs were purified from the primary culture using a previously described preplate isolation method $(\mathrm{Qu}$ et al., 1998). In brief, hindlimb muscles of mice were removed and minced into a coarse slurry using scalpels. The muscle tissues were enzymatically dissociated in collagenase type XI (Sigma, St. Louis, MO), dispase (Invitrogen, Carlsbad, CA), and trypsin-EDTA (Invitrogen). After the physical and enzymatic dissociations, muscle-derived cells were centrifuged and resuspended in proliferation medium (PM) containing DMEM, 10\% horse serum, $10 \%$ FBS, $1 \%$ chick embryo extract and $1 \%$ penicillin-streptomycin. To isolate SMNPs, the cells were plated in collagen-coated flasks (PP1) (Figure 1A). After $2 \mathrm{~h}$, cells non-adherent in PP1 were transferred into the next flasks (PP2) and incubated for $16 \mathrm{~h}$. This procedure was continued for a further three passages (PP3 through PP5) made at indicated time intervals. Since no adherent cells were observed in PP5, the serial transfer of non-adherent cells was terminated at this point. During cultivation, two morphologically different cell populations were identified. These were fibroblast-like cells and round-shaped cells. The PP1 flasks initially contained a high proportion of fibroblast-like cells. The PP2, PP3, and PP4 flasks contained approximately $30 \%, 10 \%$, and < $1 \%$ of fibroblast-like cells, respectively. Further purification of the weakly adherent cells (primarily round-shaped cells) was performed on cells adherent in the PP3 and PP4 flasks. The cells were trypsinized for $3 \mathrm{~min}$, after which the suspended cells from the PP3 and PP4 flasks were combined and replated in a fresh flask. As these cells proliferated in PM for 3-4 d, the cells formed additional colonies. The colony-forming cell clones were re-trypsinized and replated in fresh flasks. This procedure was repeated at 3-4 d intervals for 2-3 wk. After the total cell number attained $1 \times$ $10^{6}$, we named these cells "SMNPs". The SMNPs were characterized by immunocytochemistry and RT-PCR analysis for several (stem) cell markers (Figure 1B).

\section{Neuronal induction}

The SMNPs were seeded on laminin-coated sixwell plates or coverslips. The cells were then placed into one of three types of neural induction media. These were DFN [DMEM/F12 containing 2 $\mathrm{mM}$ L-glutamine, and N2 (Invitrogen)], RA + DFN [DFN with $1.5 \mu \mathrm{M}$ all-trans retinoic acid (RA, Sigma)], and FSK + DFN [DFN with $10 \mu \mathrm{M}$ forskolin (FSK; Tocris Bioscience, Ellisville, Mo)]. After $5 \mathrm{~d}$, the cells were characterized by immunocytochemistry, RT-PCR, and Western blot analysis (Figure 1B).

\section{Immunocytochemistry}

The cells were fixed in $4 \%$ paraformaldehyde for 
20 min. Cells were then covered with a solution containing $0.1 \%$ Triton $\mathrm{X}-100,0.1 \%$ normal serum, and a primary antibody. Cells were incubated at $4^{\circ} \mathrm{C}$ overnight. The primary antibodies used were rabbit anti-sox2 (1:100; Chemicon, Temecula, CA), mouse anti-CD34 (1:200; BD Pharmingen, San Diego, CA), mouse anti-CD45 (1:200; BD Transduction Laboratories, Palo Alto, CA), mouse antidesmin (1:200; BD Pharmingen), mouse anti- $\beta$ III tubulin (1:500; Covance Research Products Inc., Richmond, CA) and mouse anti-nestin (1:500; Developmental Studies Hybridoma Bank, lowa City, IA). Immunoreactivity was visualized using the antibodies Alexa Fluor 488- or 568-conjugated anti-mouse IgG (1:500; Invitrogen), or Alexa Fluor 568-conjugated anti-rabbit IgG (1:500; Invitrogen). Cells were counterstained with DAPI (Vector Laboratories, Burlingame, CA) to visualize cell nuclei.

\section{RNA extraction and RT-PCR analysis}

Total RNA was isolated from cells using the RNABee system (TEL-TEST Inc., Friendwood, TX). Isolated RNA $(1 \mu \mathrm{g})$ was subjected to reverse transcription using the Accupower RT premix
(Bioneer, Daejeon, Korea). PCR amplification was carried out using sequence-specific primers. The specific primer sequences and expected PCR product sizes were: nestin (5'-CAAGAACCACTGGGGTCTGT-3', 5'-ACATCCTCCCACCTCTGTTG3'; 253 bp), notch1 (5'-CGGTGTGAGGGTGATG TCAATG-3', 5'-GAATGTCCGGGCCAGCGCCACC-3'; 534 bp), neuroD (5'-ACGCAGAAGGCAAGGTGT-3', 5'-GCCTTCATGCGCCTTAATTT-3'; 399 bp), nse (5'-TTGGAGCTGGTGAAGGAAGCC-3', 5'-CCTTGAGCAGCAAACAGTTGC-3'; 360 bp), sox2 (5'-TTCGGTGATGCCGACTAGA-3', 5'-TGCGAAGCGCCTAACGTA-3'; 100 bp), egfR (5'-AAGTGGTCCTTGGGAACTTG-3', 5'-TTGAGGGCAATGAGGACATA-3'; 106 bp), calretinin (5'-GGAGATGAACATCCAACAGC-3', 5'-TCACTGCAGAGCACAATCTC-3'; 105 bp), syn1 (5'-GACGAGGTGAAAGCTGAGAC-3', 5'-CAGAGAGGGCTGTCTAGGG-3'; 110 bp), tau (5'-TAGCAACGTCCAGTCCAAGT-3', 5'-GTCACTTTGCTCAGGTCCAC-3'; 105 bp), $\alpha$-sma (5'-ACCACCATGTACCCTGGTAT-3', 5'AGCACGATTGTCGATTGTCG-3'; 280 bp) and gapdh (5'-TCCATGACAACTTTGGCATCGTGG-3', 5'-GTTGCTGTTGAAGTCACAGGAGAC-3'; 376 bp). PCR products were separated on agarose gels and visualized by ethidium bromide staining.
A
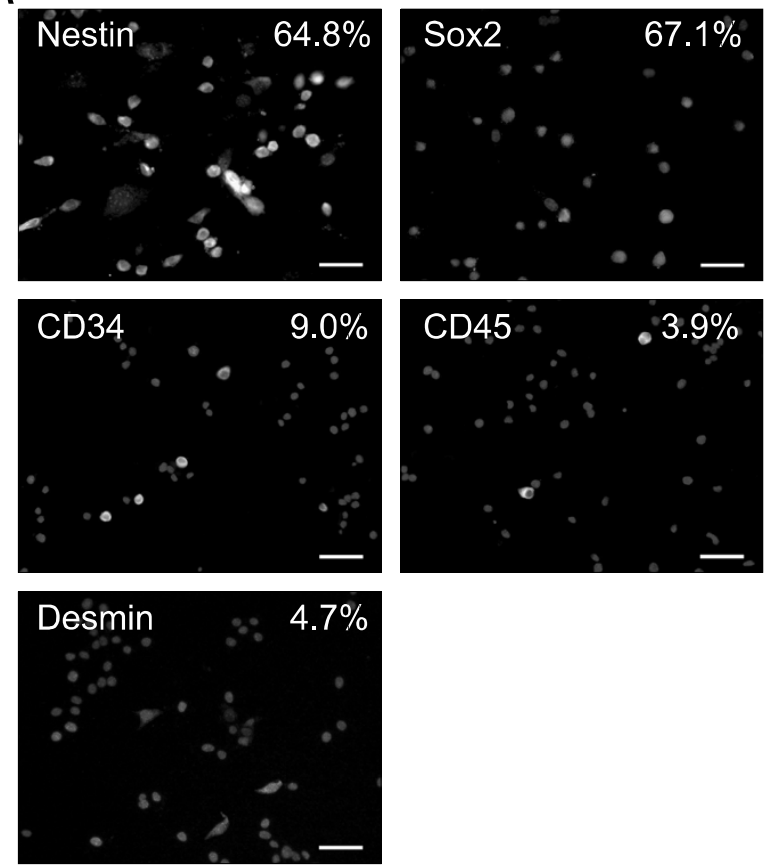

B
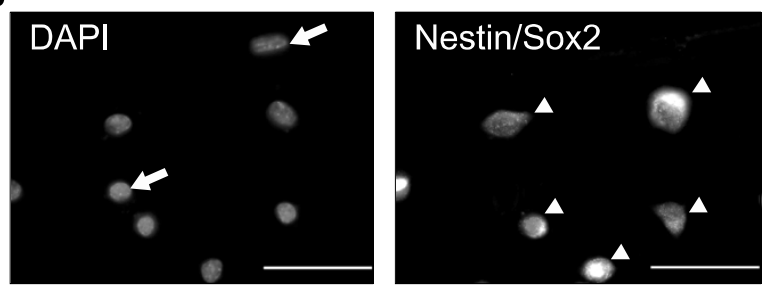

C

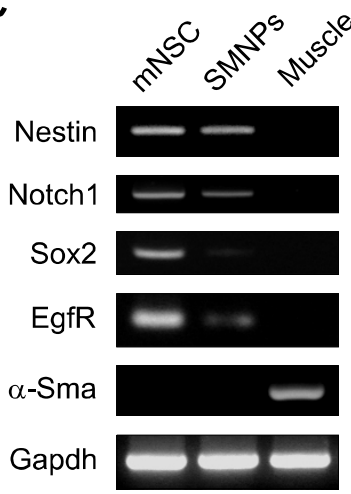

Figure 2. Characterization of SMNPs. (A) The characteristics of SMNPs were determined by immunocytochemistry using neural stem cell markers (nestin and sox2), hematopoietic stem cell markers (CD34 and CD45), and a myogenic cell marker (desmin). The percentages shown represent the proportions of marker-positive cells. (B) Nestin immunofluorescence is merged with sox2 immunofluorescence. DAPI staining shows cell nuclei in the same field. The arrowheads indicate nestin and sox2 double-positive cells. The arrows point to negative cells for nestin and sox2. (C) The characteristics of SMNPs were determined by RT-PCR using NSC and muscle markers $(\alpha$-sma). gapdh was used as the internal control gene. mNSC, mouse neural stem cell. Scale bar: $50 \mu \mathrm{m}$. 
Quantitative analysis of DNA bands was performed with imaging software (Gene Tools; Sygene, UK).

\section{Western blot analysis}

Cells were washed with ice-cold PBS (10 mM $\mathrm{Na}_{2} \mathrm{HPO}_{4}, 2 \mathrm{mM} \mathrm{KH} \mathrm{PO}_{4}, 137 \mathrm{mM} \mathrm{NaCl}$ and 2.7 $\mathrm{mM} \mathrm{KCl}$ ) and lysed with RIPA buffer (50 mM Tris- $\mathrm{HCl} \mathrm{pH} \mathrm{7.4,} 150 \mathrm{mM} \mathrm{NaCl}, 1 \%$ Triton X-100, $1 \%$ sodium deoxycholate, $0.1 \%$ SDS, 2 mM EDTA, and protease inhibitors). Cell lysates were centrifuged at $12,000 \mathrm{rpm}$ for $10 \mathrm{~min}$. Fifty micrograms of protein samples were mixed with SDS sample buffer, boiled for $5 \mathrm{~min}$, and then subjected to $9 \%$ SDS-PAGE before being electrotransferred to a PVDF membrane (Schleicher \& Schuell Bioscience, Keene, $\mathrm{NH}$ ). The membrane was blocked with $5 \%$ skim milk for $1 \mathrm{~h}$ at room temperature and incubated with mouse anti- $\beta$ III-tubulin and mouse anti- $\alpha$-tubulin antibodies (Clontech, Palo Alto, CA). The membrane was then incubated with antimouse HRP-conjugated IgG (Zymed, San Francisco, CA). Protein visualization was achieved using the Supersignal West Pico Chemilumi-

A

Neural induction
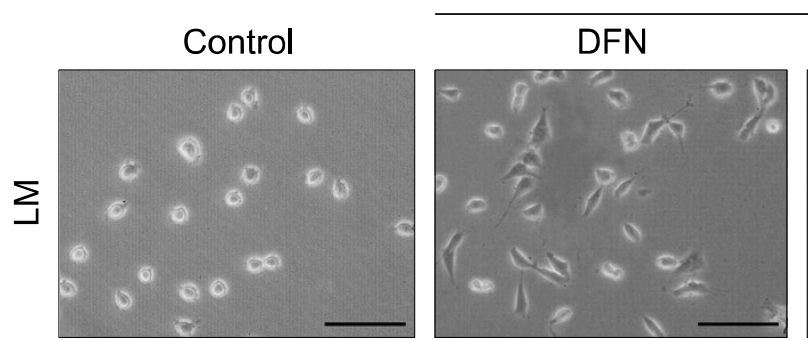

RA + DFN
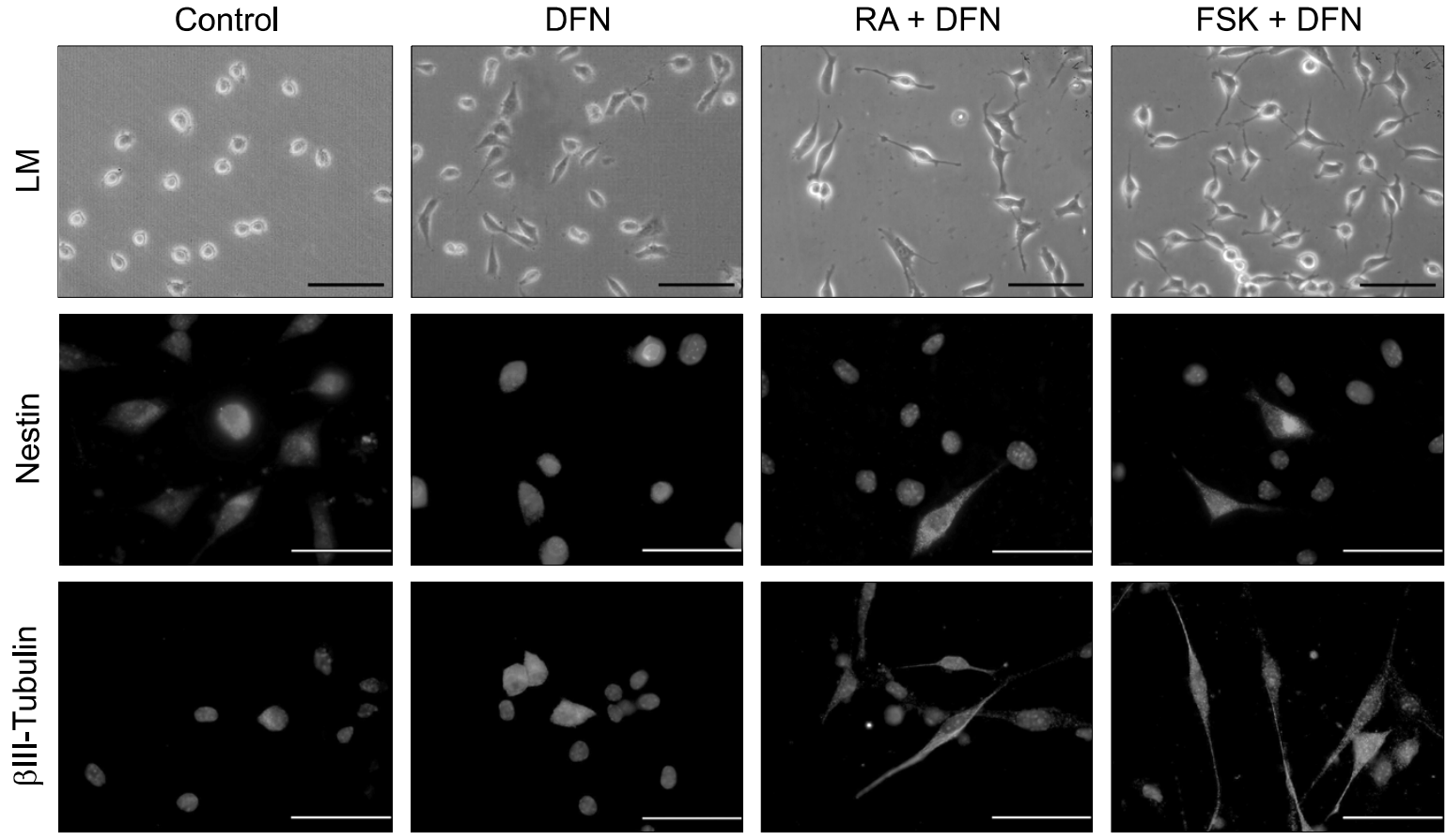

B
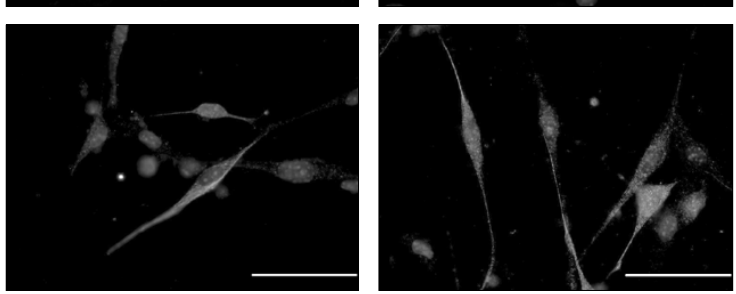

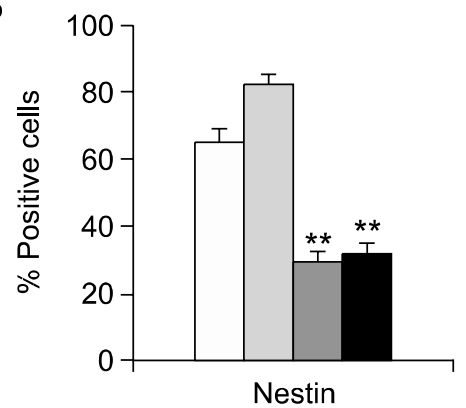

C

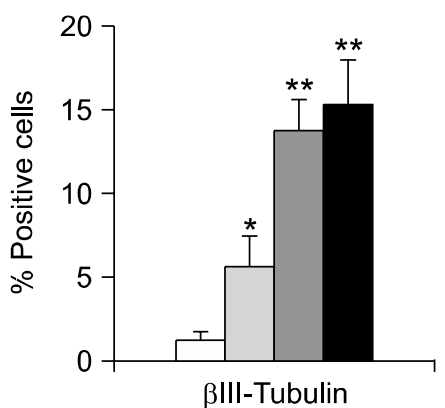

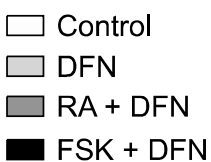

Figure 3. Immunocytochemistry of SMNPs before and after neural induction. (A) Before and after neural induction, cell morphologies were examined by light microscopy (LM). Characteristics of SMNPs were visualized by immunocytochemistry using antibodies against nestin and $\beta$ III-tubulin. DAPI staining shows cell nuclei. Immunofluorescence of markers is merged with DAPI signals. (B) The proportions of nestin-positive cells were measured before (control) and after three types of neural induction. (C) The proportions of $\beta$ Ill-tubulin-positive cells in pre-induction cells, and in cells after three types of neural induction, were measured. The data are the averages \pm SEs of three independent experiments ( $P<0.05$, ${ }^{*} P<0.001$, both vs. control). Scale bar: $50 \mu \mathrm{m}$. 
nescent Substrate (Pierce, Rockford, IL) according to the manufacturer's protocol.

\section{Results}

SMNPs were isolated by the preplate isolation method as described in the Materials and Methods section. After isolation and proliferation, the characteristics of the SMNPs were determined by immunocytochemistry using several (stem) cell markers such as neural stem cell (NSC) markers (Nestin and Sox2) (Kondo et al., 2006), hematopoietic stem cell (HSC) markers (CD34 and CD45) (Alessandri et al., 2004), and a myogenic cell marker (Desmin) (Qu-Petersen et al., 2002). Interestingly, when specific neural induction materials were not used, nestin and sox2 proteins were expressed in $64.8 \%$ and $67.1 \%$ of SMNPs, respectively, whereas small numbers of SMNPs expressed HSC and myogenic cell markers (Figure 2A). In addition, nestin and sox2 proteins were commonly co-expressed in a single cell (Figure 2B). To confirm the NSC marker expression in SMNPs, we carried out RT-PCR analysis. This showed that SMNPs constitutively expressed the nestin, notch1, sox2, and egfR genes (Figure 2C). These results indicated that the SMNPs had characteristics of neural precursor cells.

To determine whether SMNPs differentiate into cells possessing neuronal characteristics, we used three types of neural induction media, DFN, RA + DFN and FSK + DFN, as described in the Materials and Methods section. After $5 \mathrm{~d}$ of incubation with neural induction media, the SMNPs changed from round-shaped cells into process-bearing cells (Figure $3 \mathrm{~A}$ ). To evaluate the effects of the neural induction media on the neural precursor characteristics of SMNPs, we performed immunocytochemistry using an antibody against nestin, both before and after neural induction. As mentioned above, a high proportion of cells expressed nestin before neural induction (Figure $3 A$ and $B$, control). Neural induction by DFN alone still resulted in a high proportion of nestin-positive cells. Additional treatment with RA or FSK decreased the percentages of nestin-positive cells by $44.5 \%$ or $48.9 \%$ respectively, compared to control. To determine if incubation of SMNPs in neural induction media might induce the expression of neuronal markers, the levels of $\beta$ III-tubulin-positive cells were measured (Kim and Son, 2006). Neural induction by DFN alone, by RA + DFN, or by FSK + DFN, increased the levels of $\beta$ Ill-tubulin-positive cells 4.5-, 11.0- and 12.3-fold, respectively, compared to controls (Figure $3 A$ and $C$ ). Glial cell markers were not detectable after any neural induction method. Taken together, these results show that treatment with RA or FSK, in DFN, significantly decreased the potency as the neural precursor cell and dramatically increased the expression of neuronal marker.

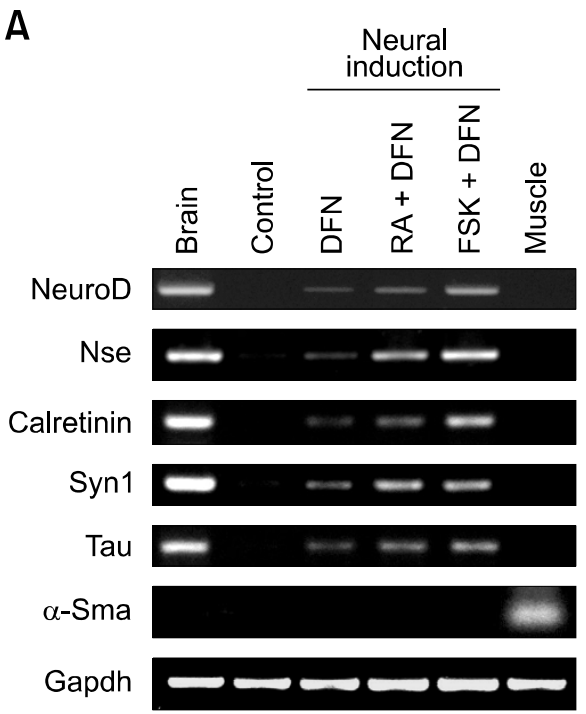

B

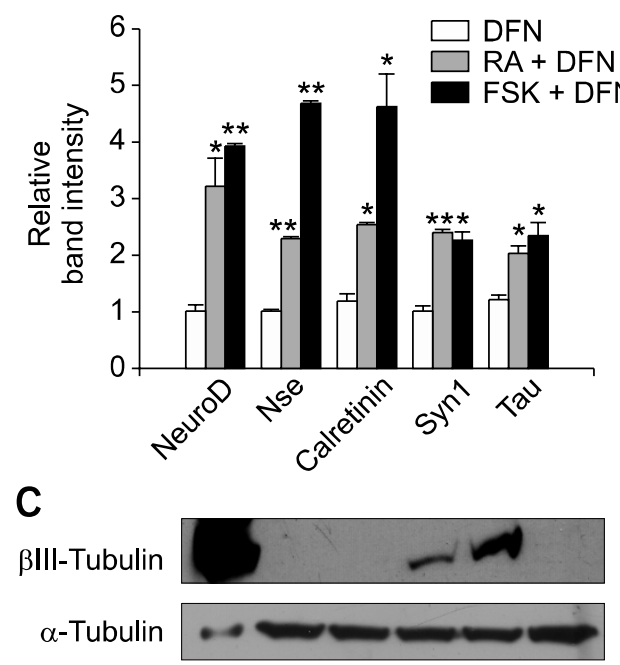

Figure 4. RT-PCR and Western blot analysis of SMNPs before and after neural induction. (A) Before and after neural induction, the expression of neuronal and muscle markers was determined by RT-PCR analysis. gapdh was used as an internal control. Brain and muscle were used as a positive and negative control, respectively. (B) Relative band intensities after various methods of neural induction are quantified by densitometry. The data are the averages \pm SEs of three independent experiments $\left({ }^{*} P<0.05,{ }^{* *} P<0.001\right.$, both vs. DFN alone). (C) Before and after neural induction, the expression of $\beta$ III-tubulin protein was measured by Western blot analysis. $\alpha$-tubulin was used as an internal control. 
To verify the increased expression of neuronal marker under neural induction conditions, we carried out RT-PCR analysis. Treatment with RA or FSK, together with DFN, guided the SMNPs to express the postmitotic neuron-specific basic helix-loop-helix (bHLH) transcription factor, neuroD gene; neuron-specific enolase (nse) gene, and the neuronal subtype marker, calretinin gene. Also, the postsynaptic marker syn1 gene and the neuronal marker tau gene were expressed (Figure 4A and $B)$. The neuronal markers examined were undetectable, or present at low levels, in SMNPs before neural induction (control) (Figure 4A). Moreover, the use of RA or FSK in DFN increased the expression level of $\beta$ III-tubulin (Figure $4 C$ ).

\section{Discussion}

In this study, we used the preplate isolation method to isolate SMNPs, which could then differentiate into cells possessing neuronal characteristics when appropriate neural induction conditions were used. Qu-Petersen et al. (2002) reported that three populations of cells were isolated from skeletal muscle using the preplate isolation method. Of these, a population termed muscle-derived stem cells (MDSCs) was isolated from a small population of long-time proliferating cells. These cells formed colonies, could divide for more than 30 passages, and showed neural-lineage cell phenotypes after treatment with nerve growth factors. The characteristics of SMNPs are similar to those of MDSCs in that isolated SMNPs grew into colonies (Figure 1), showed long-time proliferation (more than 30 passages, data not shown), and could differentiate into cells possessing neuronal characteristics. Isolated MDSCs were highly positive $(79 \%)$, however, for the CD34 antigen. In contrast, only $9.0 \%$ of SMNPs were positive for CD34 (Figure 2B). The difference in CD34 expression in MDSCs compared to SMNPs suggests that the cell characteristics of SMNPs are similar, but not identical, to those of MDSCs.

In isolated SMNPs, > $64 \%$ of cells expressed the NSC markers nestin and sox2. In contrast, only a small number of these cells expressed HSC markers (Figure 2A). Recently, Kondo et al. (2006) reported that skeletal muscle-derived progenitor cells isolated by flow cytometry, with specific cell sorting, constitutively expressed NSC markers including nestin, sox2, egfR, fgfR1, and notch1, but did not express HSC markers including $C D 45$, $S C L$, and PU.1. Although the isolation method of Kondo et al. (2006) was different from ours, the expression profile of stem cell markers in their cells is consistent with our data (Figure $2 \mathrm{~A}$ and $\mathrm{C}$ ). The preplate isolation method may thus be useful for the isolation of HSC-negative neural precursor cells from skeletal muscle tissues.

In this study, we used RA and FSK as neural induction materials. RA plays crucial roles in neuronal differentiation in many vertebrates (MorrissKay and Sokolova, 1996; Corcoran and Maden, 1999; Maden, 2002). FSK, an adenylyl cyclase activator that increases intracellular cAMP, can also induce neuronal differentiation in several neuroblastoma (Ghil et al., 2000; Kim et al., 2004) as well as in stem cells including NSCs (Ray and Gage, 2006), MSCs (Kim et al., 2005), and olfactory neuroepithelial-derived progenitors (Zhang et al., 2006). Most studies on the neuronal differentiation of skeletal muscle-derived cells have used media containing various growth factors, neurotrophic factors, and hormones, as neural induction materials (Romero-Ramos et al., 2002; Alessandri et al., 2004; Kondo et al., 2006). We, however, used only RA or FSK in a neurobasal medium. Although no other factors (growth factors, neurotrophic factors or hormones) were added, the expression of neuronal markers dramatically increased. These results suggest that both RA and FSK are powerful neuronal inducers of SMNPs.

Primary central nervous system-derived embryonic or adult NSCs are generally cultured in DFN with growth factors such as bFGF or EGF. When growth factors are withdrawn, enriched nestin positive-NSCs exit the cell cycle and start to differentiate into various neural lineage cells. In this study, although the expression of neuronal makers was increased in DFN alone (no growth factors or serum were present), SMNPs still showed high nestin expression levels. This implies, first, that the characteristics of SMNPs are different from those of NSC, and, second, that withdrawal of growth factors or serum is not sufficient to initiate differentiation into neuron-like cells. SMNPs require neural induction supporters such as RA or FSK for neuron-like cell differentiation.

The ability of neuroD, one of the proneural bHLH transcription factors, to regulate neuronal differentiation, has been noted in several systems. For example, ectopic expression of neuroD in Xenopus oocytes led to premature differentiation of neurons and conversion of a subset of presumptive epidermal cells into neurons (Lee et al., 1995). In neuroD null mice, glial differentiation of retinal explants was enhanced (Morrow et al., 1999). In addition, overexpression of neuroD induced neuronal differentiation of neuroblastoma cells (Cho et al., 2001). Similarly, expression of neuroD in cultured P19 cells promoted neuronal differentia- 
tion (Farah et al., 2000). Taken together, these data suggest that neuroD promotes the development of neurons. In this study, neural induction of SMNPs with RA + DFN or FSK + DFN strongly promoted the expression of neuroD gene, which likely subsequently induces maturation of neurons. Indeed, treatment with RA or FSK facilitated the expression of a matured or differentiated neuronal marker, nse gene (Figure 4A and B). These data imply that RA or FSK are key factors for the differentiation of SMNPs into neuron-like cells.

It is of particular interest that differentiation into glial cells was not detectable under any neural induction condition tested. This may be because 1) SMNPs, unlike NSCs, do not contain glial precursor cells, or, 2) neither RA nor FSK induces glial cell differentiation from SMNPs. Astrocytes are known to require more factors (such as ciliary neurotrophic factor and leukemia inhibitory factor) for differentiation and maintenance than do neurons (Bonni et al., 1997; Rajan and Mckay, 1998). Factors other than RA or FSK may be required for differentiation of glial cells from SMNPs. Although isolated SMNPs have neurogenic potential, most differentiated SMNPs were still of unidentified cell types.

After additional incubation in neural induction media for more than $5 \mathrm{~d}$, SMNPs, by now almost completely differentiated, were died. Several explanations may be offered. First, neuronal cells required for synapse formation and constant uptake of neurotransmitters or cytokines from the other neuronal cells. SMNPs might fail, however, to receive such stimuli from other neuronal cells. Another possibility is that glial cell differentiation may be poor under the neural induction conditions tested. Since glia contributes to brain function mainly by insulating, supporting, and nourishing neighboring neurons, the absence of glia may cause critical problems to survival of neuron.

In this study, two major issues were addressed. We found, first, that SMNPs isolated by the preplate isolation method constitutively expressed NSC-specific markers, and, second, that treatment with RA or FSK induced SMNPs to become process-bearing cells, and to express neuron- specific markers. These findings will be valuable when SMNPs are to be used for autologous transplantation in patients requiring for need of new neuronal cells, or as gene transduction carriers in future gene- and cell-based therapies for neuronal diseases.

\section{Acknowledgement}

This work was supported by the Korea Science and
Engineering Foundation (KOSEF) grant funded by the Korea government (MOST) (grant No. R01-2004-00010622-0), the Neurobiology Research Program from the KOSEF (grant No. M10412000017-06N1200-01710), and 2006 Kyonggi University Specialization Program.

\section{References}

Alessandri G, Pagano S, Bez A, Benetti A, Pozzi S, lannolo G, Baronio M, Invernici G, Caruso A, Muneretto C, Bisleri G, Parati $E$. Isolation and culture of human muscle-derived stem cells able to differentiate into myogenic and neurogenic cell lineages. Lancet 2004;364:1872-83

Bonni A, Sun $Y$, Nadal-Vicens M, Bhatt A, Frank DA, Rozovsky I, Stahl N, Yancopoulos GD, Greenberg ME. Regulation of gliogenesis in the central nervous system by the JAK-STAT signaling pathway. Science 1997;278:477-83

Cho JH, Kwon IS, Kim S, Ghil SH, Tsai MJ, Kim YS, Lee YD, Suh-Kim H. Overexpression of BETA2/NeuroD induces neurite outgrowth in F11 neuroblastoma cells. J Neurochem 2001;77:103-9

Corcoran J, Maden M. Nerve growth factor acts via retinoic acid synthesis to stimulate neurite outgrowth. Nat Neurosci 1999;2:307-8

Farah MH, Olson JM, Sucic HB, Hume RI, Tapscott SJ, Turner DL. Generation of neurons by transient expression of neural bHLH proteins in mammalian cells. Development 2000;127:693-702

Ghil SH, Kim BJ, Lee YD, Suh-Kim H. Neurite outgrowth induced by cyclic AMP can be modulated by the alpha subunit of Go. J Neurochem 2000;74:151-8

Hwang JH, Yuk SH, Lee JH, Lyoo WS, Ghil SH, Lee SS, Khang IG, Paik SY, Lee JY. Isolation of muscle derived stem cells from rat and its smooth muscle differentiation. Mol Cells 2004; 17:381

Jiang Y, Vaessen B, Lenvik T, Blackstad M, Reyes M, Verfaillie CM. Multipotent progenitor cells can be isolated from postnatal murine bone marrow, muscle, and brain. Exp Hematol 2002;30:896-904

Kim BJ, Kim SS, Kim YI, Paek SH, Lee YD, Suh-Kim H. Forskolin promotes astroglial differentiation of human central neurocytoma cells. Exp Mol Med 2004;36:52-6

Kim BW, Son H. Neural cell adhesion molecule (NCAM) induces neuronal phenotype acquisition in dominant negative MEK1-expressing hippocampal neural progenitor cells. Exp Mol Med 2006;38:732-8

Kim SS, Choi JM, Kim JW, Ham DS, Ghil SH, Kim MK, Kim-Kwon Y, Hong SY, Ahn SC, Kim SU, Lee YD, Suh-Kim $\mathrm{H}$. cAMP induces neuronal differentiation of mesenchymal stem cells via activation of extracellular signal-regulated kinase/MAPK. Neuroreport 2005;16:1357-61

Kondo T, Case J, Srour EF, Hashino E. Skeletal musclederived progenitor cells exhibit neural competence. Neuroreport 2006;17:1-4

Lee JE, Hollenberg SM, Snider L, Turner DL, Lipnick N, 
Weintraub H. Conversion of Xenopus ectoderm into neurons by NeuroD, a basic helix-loop-helix protein. Science 1995; 268:836-44

Lin Y, Luo E, Chen X, Liu L, Qiao J, Yan Z, Li Z, Tang W, Zheng $X$, Tian W. Molecular and cellular characterization during chondrogenic differentiation of adipose tissue-derived stromal cells in vitro and cartilage formation in vivo. J Cell Mol Med 2005;9:929-39

Maden M. Retinoid signalling in the development of the central nervous system. Nat Rev Neurosci 2002;3:843-53

McKinney-Freeman SL, Jackson KA, Camargo FD, Ferrari G, Mavilio F, Goodell MA. Muscle-derived hematopoietic stem cells are hematopoietic in origin. Proc Natl Acad Sci USA 2002;99:1341-6

Morriss-Kay GM, Sokolova N. Embryonic development and pattern formation. FASEB J 1996;10:961-8

Morrow EM, Furukawa T, Lee JE, Cepko CL. NeuroD regulates multiple functions in the developing neural retina in rodent. Development 1999;126:23-36

Muguruma Y, Reyes M, Nakamura Y, Sato T, Matsuzawa $\mathrm{H}$, Miyatake H, Akatsuka A, Itoh J, Yahata T, Ando K, Kato S, Hotta T. In vivo and in vitro differentiation of myocytes from human bone marrow-derived multipotent progenitor cells. Exp Hematol 2003;31:1323-30

Peng $\mathrm{H}$, Huard J. Muscle-derived stem cells for musculoskeletal tissue regeneration and repair. Transpl Immunol 2004;12:311-9

Qu-Petersen Z, Deasy B, Jankowski R, Ikezawa M, Cummins J, Pruchnic R, Mytinger J, Cao B, Gates C, Wernig A, Huard J. Identification of a novel population of muscle stem cells in mice: potential for muscle regeneration. J Cell Biol 2002;157:851-64
Qu Z, Balkir L, van Deutekom JC, Robbins PD, Pruchnic R, Huard J. Development of approaches to improve cell survival in myoblast transfer therapy. J Cell Biol 1998;142:1257-67

Rajan P, McKay RD. Multiple routes to astrocytic differentiation in the CNS. J Neurosci 1998;18:3620-9

Ray J, Gage FH. Differential properties of adult rat and mouse brain-derived neural stem/progenitor cells. Mol Cell Neurosci 2006;31:560-73

Romero-Ramos M, Vourc'h P, Young HE, Lucas PA, Wu Y, Chivatakarn O, Zaman R, Dunkelman N, el-Kalay MA, Chesselet MF. Neuronal differentiation of stem cells isolated from adult muscle. J Neurosci Res 2002;69:894-907

Sun JS, Wu SY, Lin FH. The role of muscle-derived stem cells in bone tissue engineering. Biomaterials 2005;26:3953-60

Toma JG, Akhavan M, Fernandes KJ, Barnabe-Heider F, Sadikot A, Kaplan DR, Miller FD. Isolation of multipotent adult stem cells from the dermis of mammalian skin. Nat Cell Biol 2001;3:778-84

Toma JG, McKenzie IA, Bagli D, Miller FD. Isolation and characterization of multipotent skin-derived precursors from human skin. Stem Cells 2005;23:727-37

Wada MR, Inagawa-Ogashiwa M, Shimizu S, Yasumoto S, Hashimoto N. Generation of different fates from multipotent muscle stem cells. Development 2002;129:2987-95

Zhang X, Klueber KM, Guo Z, Cai J, Lu C, Winstead WI, Qiu $M$, Roisen FJ. Induction of neuronal differentiation of adult human olfactory neuroepithelial-derived progenitors. Brain Res 2006;1073-1074:109-19

Zuk PA, Zhu M, Ashjian P, De Ugarte DA, Huang JI, Mizuno $\mathrm{H}$, Alfonso ZC, Fraser JK, Benhaim P, Hedrick MH. Human adipose tissue is a source of multipotent stem cells. Mol Biol Cell 2002;13:4279-95 